\title{
Carrier Class Availability Prediction for Hybrid FSO/RF System in Heavy Rainfall Regions Based on ITU-R Models
}

\author{
Ahmed Basahel and Wajdi Al-Khateeb
}

\begin{abstract}
Availability is considered as the main parameter of evaluating a Hybrid FSO/RF link quality. An accurate carrier class availability prediction of Hybrid FSO/RF is needed. In tropical regions, among different weather influences, rain plays a major role. Precipitation decrees are available for frequencies above $10 \mathrm{GHz}$. In this paper we will analyze the effect of rain on Hybrid FSO/RF to find the optimum operating frequency to back-up the FSO link besides tradeoff between speed of RF and link availability of FSO in tropical environment; we will also provide better prediction of link availability. ITU-R specific rain attenuation of FSO and RF models has been used for the analysis. From the results, a $30 \mathrm{GHz}$ and below are suggested to be used as a back-up of FSO link to achieve carrier grade availability under the impact of rain.
\end{abstract}

Index Terms-Carrier grade availability, heavy rain attenuation hybrid FSO/RF, ITU-R specific rain attenuation models.

\section{INTRODUCTION}

Free Space Optics is a wireless technology which uses laser as a medium of transmission between transmitter and receiver. This technology is a line of sight (LOS) where transmitter and receiver should be on mountain or at the roof top of buildings.

FSO has many advantages such as high bandwidth, easy to install where you can install transmitter and receiver beside a window and within days, free licensing of frequency, cost effectiveness where no digging is needed and very high security where the signal cannot be hacked.

The big challenge facing this technology and considered a disadvantage is that FSO especially in long distances can easily be affected by weather conditions like fog, haze, rain and scintillation [1], [2]. In temperate regions, fog is consider the main factor which influences the performance of FSO link availability [3], [4]; whereas in tropical regions heavy rainfall rate is expected to be the limiting factor for FSO link availability. These weather conditions limit the distance of FSO and decrease the link availability [5]. In order to compensate this problem we need to have a comprehensive FSO system; one of the solutions is to implement FSO link with a back-up RF (Hybrid FSO/RF system). FSO/RF system can enhance the performance of FSO system and optimize the link quality such as the availability of FSO performance; by using FSO/RF the availability can increase from $99.99 \%$

Manuscript received March 9, 2014; revised June 1, 2014.

The authors are with the Department of Electrical and Computer Engineering at IIUM, 53100 KL, Malaysia (e-mail: ba_sahal@hotmail.com, wajdi@iium.edu.my). (enterprise class) to $99.999 \%$ (carrier class) [2]. In this paper, the availability is calculated using link budget of FSO system and microwave. As the rain attenuation of Hybrid FSO/RF is independent of wavelength, the analysis will be based on rainfall rate [6]. ITU-R specific rain attenuation models for FSO and RF are used for the analysis. The effect of rain attenuation on the availability of Hybrid $\mathrm{FSO} / \mathrm{RF}$ is analyzed by examining the distance of different RF frequencies. A recommendation of optimum operating frequency for tradeoff of FSO availability is suggested.

Over the years, FSO technology has gained acceptance in telecommunication industry mostly in enterprise campus network. This paper provides recommendations to local telecom service provider about possible availability figures of carrier and enterprise class that can be useful for the deployment of Hybrid FSO/RF link as the last mile solution, back-up for fiber optic and other applications. Fig. 1 below shows the proposed approach of the link availability prediction used in this paper.

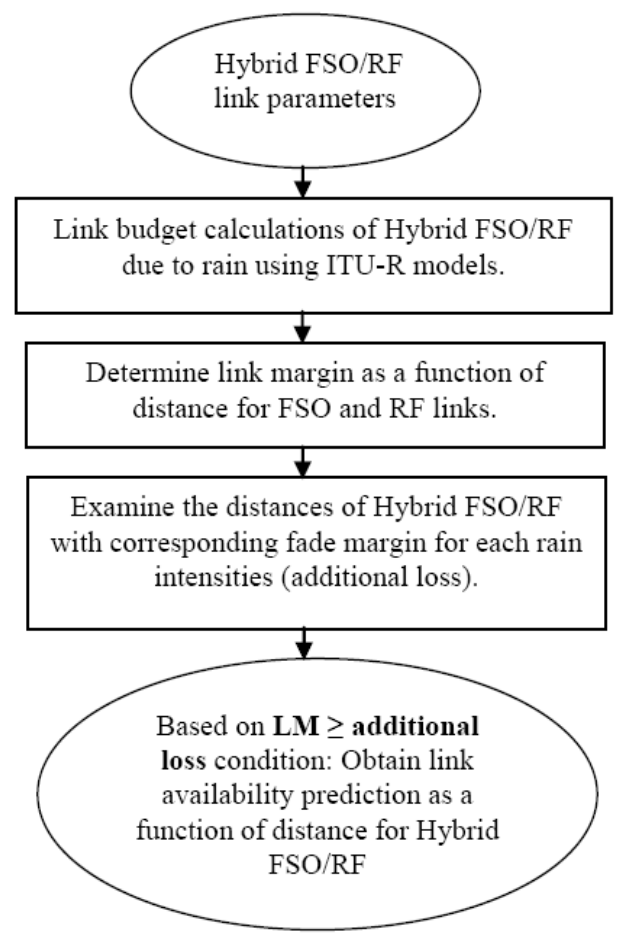

Fig. 1. Flow chart of proposed approach.

\section{FSO LINK BUDGET}

\section{A. Geometric Loss}

Light beam is expanding as it travels from point of origin outwards. The beam usually adopts a cone shape and a 
divergence angle determines how much the beam spreads as it travels through air. Thus, this will lead to the fact that a big portion of the light will miss the receiver aperture at the receiver side and will be considered as a loss. This loss is referred to as a geometric loss or attenuation. Geometric attenuation has three main parameters which can help in optimizing the performance of FSO link which are minimizing divergence angle and link distance, increasing the receiver aperture area. The divergence angle and receiver aperture area are related to the design of FSO.

Mathematically, geometrical attenuation can simply be derived from Fig. 2 below. The portion of light which misses the receiver aperture is shown.

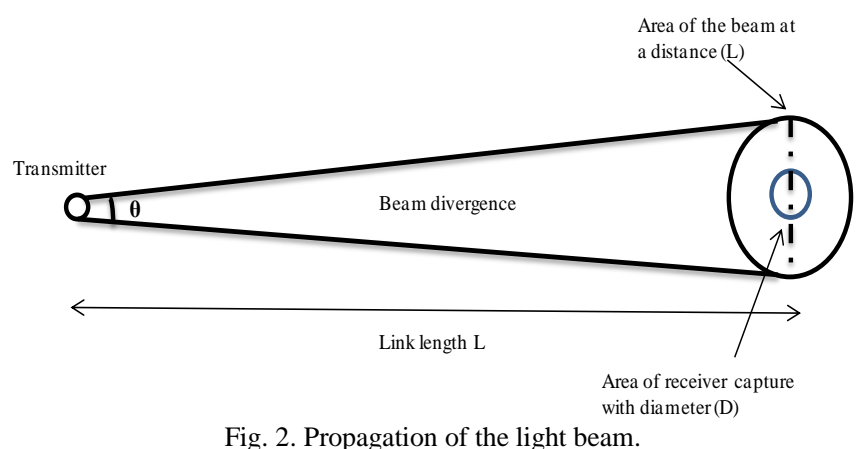

GeomAtt $=\frac{\text { Area of the beam at a distance }(L)}{\text { Area of receiver capture with diameter }(D)}$

So, simply by dividing these two circle areas as:

$$
=\frac{\pi\left(\frac{L \theta}{2}\right)^{2}}{\pi\left(\frac{D}{2}\right)^{2}}=\frac{\frac{\pi}{4}(L \theta)^{2}}{\frac{\pi}{4}(D)^{2}}=\left(\frac{L \theta}{D}\right)^{2}
$$

The geometric attenuation can be in $d B$ as follows:

$$
\begin{aligned}
& \text { GeomAtt }(d B)=10 \log \left(\frac{L \theta}{D}\right)^{2} \\
& \text { GeomAtt }(d B)=20 \log \frac{L \theta}{D}
\end{aligned}
$$

\section{B. Rain Attenuation}

In regions with heavy rainfall rates such as tropical areas, rain is the main atmospheric attenuation parameter. One of the models which estimates attenuation due to rain for FSO links in tropical areas is:

$$
A_{p}=W+A_{1 \mathrm{~km}} \times L \times r_{1}+2(d B)
$$

$A_{p}$ is the overall rain link attenuation for $\%$ of the year for path length, $L, W$, loss due to water on the FSO transceiver window, $A_{1 \mathrm{~km}}$, the rain path attenuation, $r_{1}$, normalized reduction factor. The additional $2 d B$ is taking into account the smoke haze and scintillation effects. The above model is an experimental model proposed in Singapore by [7].

\section{FSO Link Margin}

The concept of link margin of FSO link can be shown clearly in Fig. 3. The condition of link availability of Hybrid $\mathrm{FSO} / \mathrm{RF}$ is $L M \geq$ additional loss. Also, from this condition the maximum distance of Hybrid FSO/RF can be achieved when link margin $=0$.

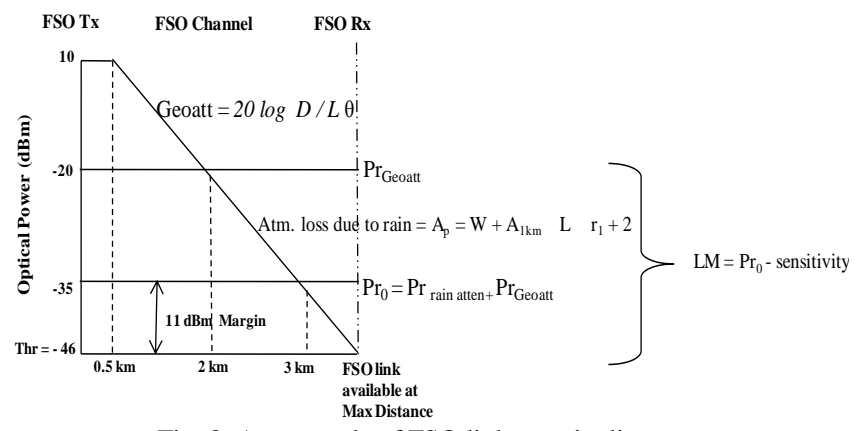

Fig. 3. An example of FSO link margin diagram.

\section{MicrowaVE LINK BUDGET}

\section{A. Free Space Loss}

The total transmission loss of a microwave link can be calculated using the following formula [8].

Attenuation $_{d B}=92.45+20 \log F_{\mathrm{GHz}}+20 \log D_{\mathrm{km}}+e_{d B}$

$e$ is excess attenuation $(d B)$ due to rainfall.

\section{B. Rain Attenuation}

Again the additional attenuation in microwave will be due to rain. The ITU-R specific attenuation model for rain of a microwave line of sight is used to determine the attenuation due to rain [9].

\section{LINK AVAILABILITY OF HYBRID FSO/RF}

The estimate availability figure calculated based on existing commercial Hybrid FSO/RF parameters is shown in Table I. In (3), ITU-R Japan model $\left(A_{1 \mathrm{~km}}=1.58 R^{0.63}\right)$ is employed for FSO channel to calculate $A_{1 \mathrm{~km}}$ parameter [10]. The reason we use this model is because of the measurement conducted to develop this model up to 80 to $90 \mathrm{~mm} / \mathrm{hr}$ rain rate which gives close figure of rain rate in heavy rainfall regions. To calculate the fade margin as a function of distance we used the rain rate of region $N$ of Table I. in ITU-R PN.837-1 with corresponding percentage of time [11]; and specific rain attenuation model of RF. The simulations have been done for many different frequencies to find the optimum operating frequency to back-up FSO link. Fig. 4 and Fig. 5 show the fade margin as a function of distance of Hybrid FSO with lower back-up frequencies $10 \& 25 \mathrm{GHz} .10 \mathrm{GHz}$ gives carrier class availability for distance more than $5 \mathrm{~km} .25 \mathrm{GHz}$ gives carrier class availability for a maximum up to $800 \mathrm{~m}$ distance.

\section{A. $\mathrm{FSO} / 10 \& 25 \mathrm{GHz}$}

The reason $10 \mathrm{GHz}$ can go up to a long distance is due to the fact that frequencies below $10 \mathrm{GHz}$ are not affected only by rain but all weather conditions, whereas above $10 \mathrm{GHz}$ are attenuated due to rainfall and atmospheric absorption. In high frequencies the wavelength will be shorter than the raindrop size causing absorption and scattering.

\begin{tabular}{lllll} 
TABLE I: HYBRID FSO/RF SYSTEMS PARAMETERS USED IN CALCULATIONS \\
\hline \hline Channels & $\begin{array}{l}\text { Transmit } \\
\text { Power }\end{array}$ & Sensitivity & $\begin{array}{l}\text { Divergence ang. } \\
\text { / Antenna Gain }\end{array}$ & $\begin{array}{l}\text { Receiver Ape. } \\
\text { / System loss }\end{array}$ \\
\hline FSO & $28 \mathrm{dBm}$ & $-34 \mathrm{dBm}$ & $2.7 \mathrm{mrad}$ & $0.2 \mathrm{~m}$ \\
RF & $26 \mathrm{dBm}$ & $-96 \mathrm{dBm}$ & $22 \mathrm{~dB}$ & $2 \mathrm{~dB}$ \\
\hline \hline
\end{tabular}




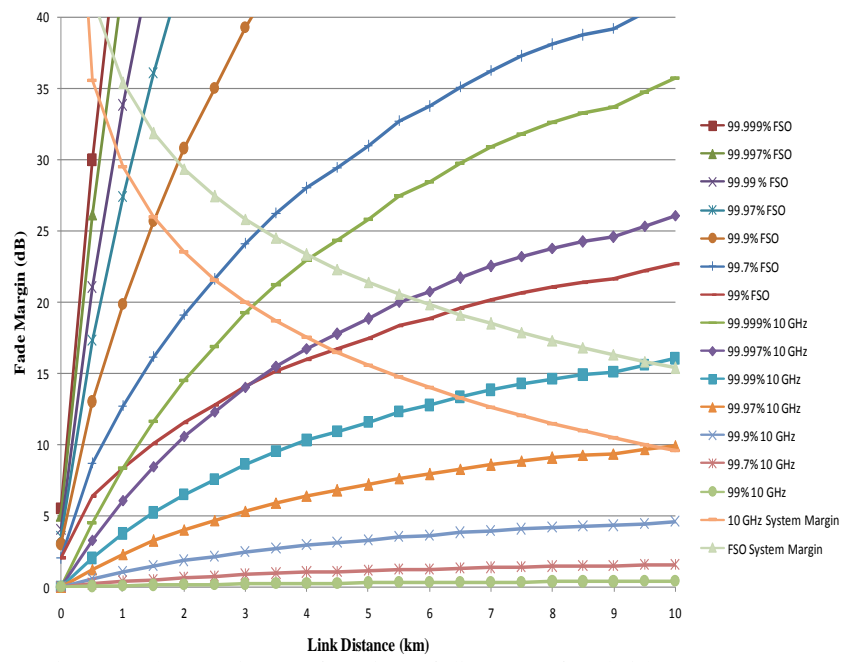

Fig. 4. Fade margin as a function of distance of Hybrid FSO/10 GHz.

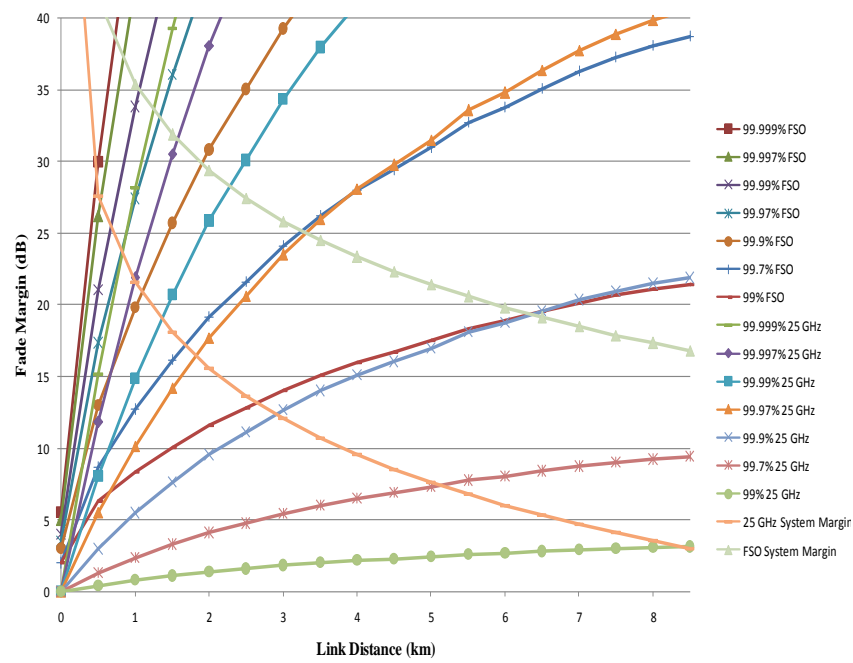

Fig. 5. Fade margin as a function of distance of Hybrid FSO/25 GHz.

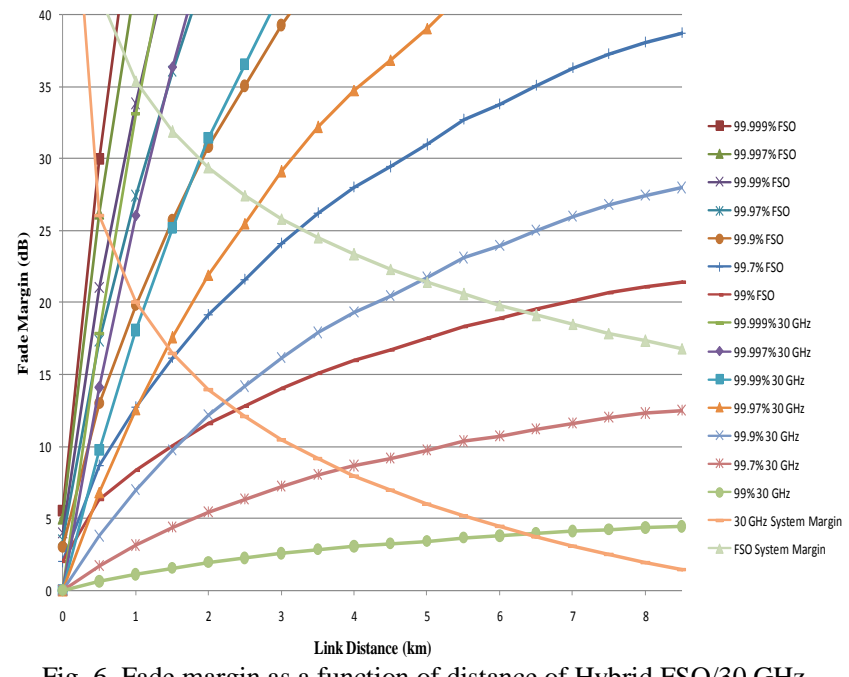

Fig. 6. Fade margin as a function of distance of Hybrid FSO/30 GHz.

\section{B. $\mathrm{FSO} / 30 \mathrm{GHz}$}

Fig. 6 shows FSO link with the optimum choice of $30 \mathrm{GHz}$ back-up. $30 \mathrm{GHz}$ can provide carrier class availability over a distance of almost $750 \mathrm{~m}$ only.

It can also provide long distance if the availability is not the priority.

\section{C. $\mathrm{FSO} / 40 \& 60 \mathrm{GHz}$}

Fig. 7 and Fig. 8 show the fade margin as a function of distance of Hybrid FSO with higher frequencies of $40 \mathrm{GHz}$ and $60 \mathrm{GHz}$. These two frequencies can operate within only a few hundred meters for carrier class availability.

Enterprise class availability for 10, 25, 30, $40 \& 60 \mathrm{GHz}$ back-up can operate over long distances as shown in Fig. 9, whereas in Fig. 10 carrier class availability with good enough resolution for Hybrid FSO/RF is shown. In the case of FSO outage, the transition or switching can be done at $30 \mathrm{GHz}$ back-up to provide carrier class availability with consideration of data rate.

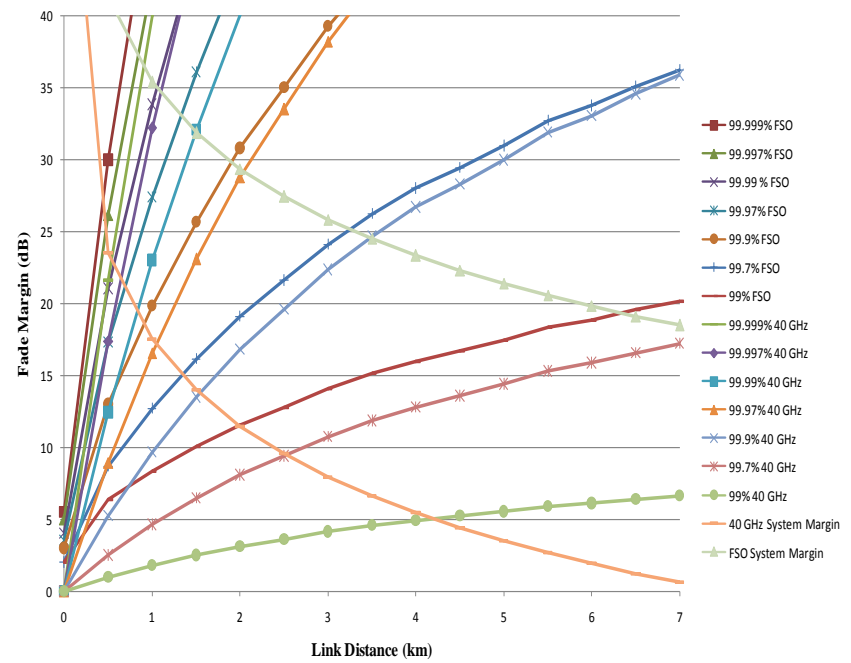

Fig. 7. Fade margin as a function of distance of Hybrid FSO/40 GHz.

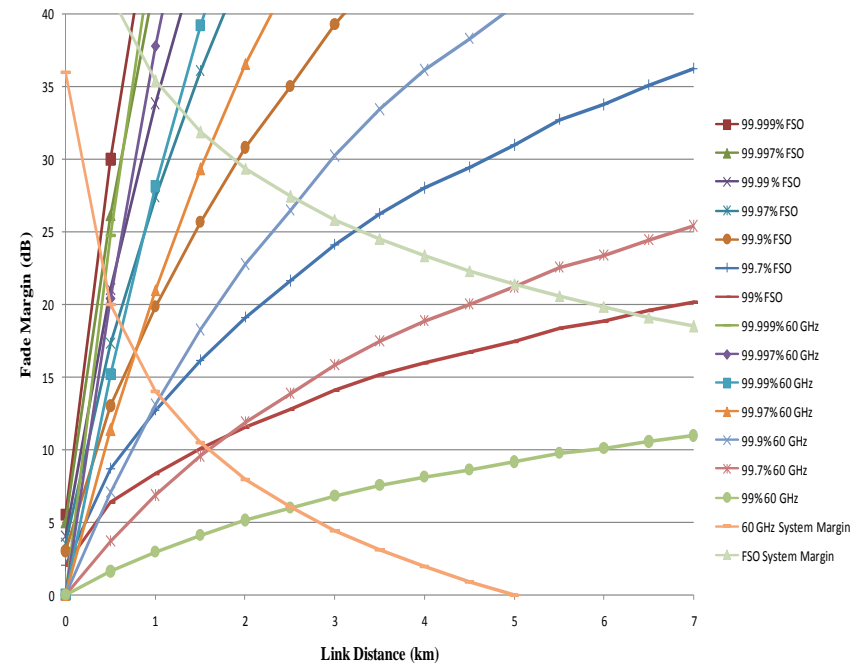

Fig. 8. Fade margin as a function of distance of Hybrid FSO/60 GHz.

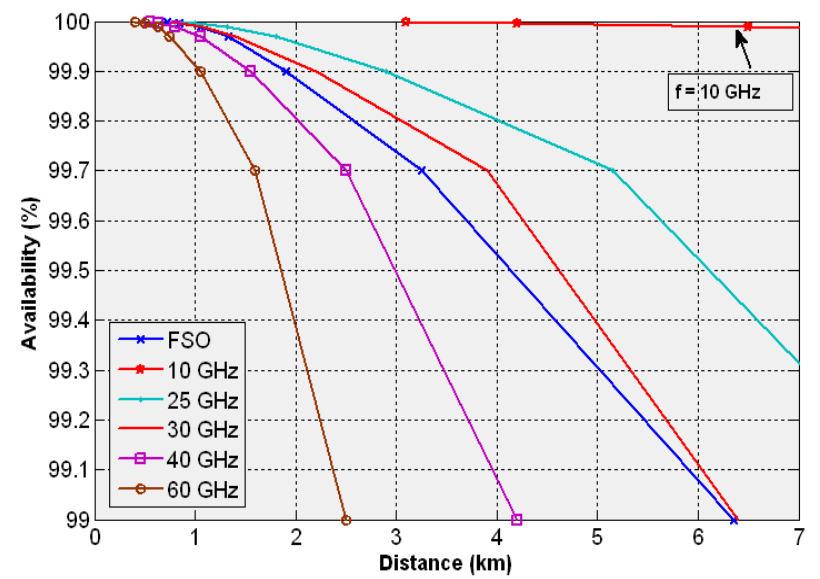

Fig. 9. Enterprise class availability of Hybrid FSO/RF. 


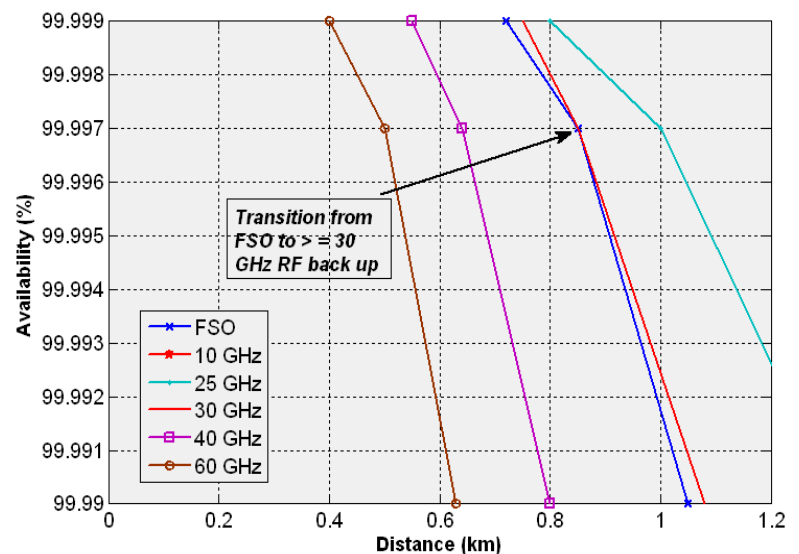

Fig. 10. Carrier class availability of Hybrid FSO/RF.

\section{CONCLUSION}

Availability in general depends on the internal parameter of Hybrid FSO/RF system such as divergence angle, receiver aperture of FSO and antenna gain of RF. Also, it depends on the weather in particular. To achieve carrier class availability of Hybrid FSO/RF under the impact of heavy rainfall climate in tropical regions, a $30 \mathrm{GHz}$ is considered the optimum operating frequency to tradeoff between speed (data rate) of RF and availability of FSO at a distance of almost $750 \mathrm{~m}$ only. In the case of enterprise class availability or data rate is not the priority; frequencies below $30 \mathrm{GHz}$ are recommended to be used for long distances (few kilometers).

\section{REFERENCES}

[1] O. Bouchet, H. Sizun, and C. Boisrobert, Free Space Optics: Propagation and Communication, 1st ed., London, UK: ISTE Ltd, 2006.

[2] I. I. Kim and E. Korevaar, "Availability of free space optics (FSO) and hybrid FSO/RF systems," SPIE. Optical Wireless Communications IV, vol. 4530, pp. 84-95, Nov 2001.

[3] F. Nadeem et al., "Weather effects on hybrid FSO/RF communication link," IEEE J. Sel. Area Commun., vol. 27, no. 9, pp. 1687-1697, December 2009.

[4] F. Nadeem, E. Letigeb, and O. Koudelka, "Comparing the rain effects on hybrid network using optical wireless and GHz Links," in Proc.
International Conference on Emerging Technologies, October 18-19, 2008, pp. 156-161.

[5] A. Prokes. (June 2009). Atmospheric effects on availability of free space optics systems. Optical Engineering. [Online]. 48(6). Available: http://spie.org/x648.html?product_id=839757.

[6] M. Achour. (April 2002). Simulating atmospheric free-space optical propagation: Part I, rainfall attenuation. Proceedings of the SPIE. [Online]. 4635.4 Available: http://proceedings.spiedigitallibrary.org/proceeding.. aspx?articleid=87 3412

[7] S. Rao, J. Ong, K. Timothy, and D. Venugopal. (June 2013). Propagation of free space optical links in Singapore. Indian Journal of Radio \& Space Physics. [Online]. 42. pp. 182-186. Available: http://nopr.niscair.res.in/bitstream/123456789/19412/1/IJRSP\%2042 \%283\%29\%20182-186.pdf.

[8] R. Freeman, Radio System Design for Telecommunications, $3^{\text {rd }}$ ed New Jersey, US: Wiley, 2007, ch. 9, p. 464.

[9] Rec. ITU-R P.838-3, Specific Attenuation Model for Rain for Use in Prediction Methods, International Telecommunication Union, 2005.

[10] Rec. ITU-R P.1814, Prediction Methods Required for the Design of Terrestrial Free-Space Optical Links, International Telecommunication Union, 2007.

[11] Rec. ITU-R P.837-1, Characteristics of Precipitation for Propagation Modeling, International Telecommunication Union, 1994.

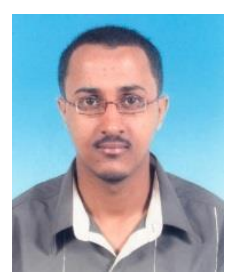

Ahmed Abdullah Basahel received his M.Sc. degree from University of Malaya (UM) 2010. He is currently a full-time PhD student at the Department of Electrical and Computer Engineering (IIUM). His research area is availability of hybrid free space optics / microwave.

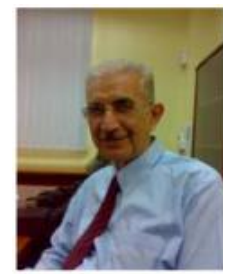

Wajdi Fawzi Al-Khateeb received his $\mathrm{PhD}$ degree from the International Islamic University Malaysia (IIUM), Malaysia 2006 and his M.Sc. degree from the Technical University of Berlin, Germany in 1968. His research interest is mainly in the reliability engineering, fault tolerant systems, QoS networking, free space optics and microwave radio links. He is currently an associate professor at the Department of Electrical and Computer Engineering, IIUM. Besides his academic activity, he was appointed as the leader of consultancy team to plan, design and supervises the ICT infrastructure project with more than 30 thousand data/voice nodes to support the ICT applications of the university. 